\title{
Cap-and-Tag Solid Phase Oligosaccharide Synthesis
}

\author{
Frédéric R. Carrel and Peter H. Seeberger \\ Laboratory of Organic Chemistry, Swiss Federal Institute of Technology (ETH) Zürich, \\ Wolfgang-Pauli-Strasse 10, 8093 Zürich, Switzerland \\ E-mail:seeberger@org.chem.ethz.ch
}

\section{Supporting Information}

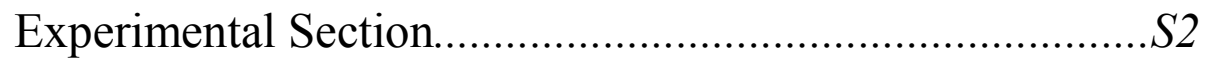

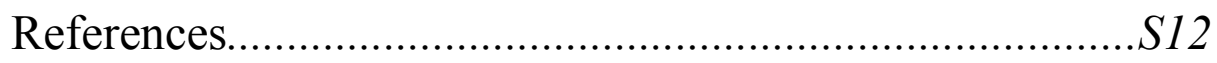


General methods. All chemicals were reagent grade and used as supplied unless mentioned otherwise. All reactions were performed in flame-dried glassware under an argon atmosphere. Dichloromethane $\left(\mathrm{CH}_{2} \mathrm{Cl}_{2}\right)$, diethylether $\left(\mathrm{Et}_{2} \mathrm{O}\right)$, tetrahydrofuran (THF) and toluene were purified by a J. C. Meyer Solvent Dispensing System (two packed columns of neutral alumina). Solvents for chromatography and work-up procedures were distilled from commercially available technical grade solvents. Analytical thin-layer chromatography was performed on E. Merck silica gel $60 \mathrm{~F}_{254}$ plates $(0.25 \mathrm{~mm})$. Compounds were visualized by $\mathrm{UV}$ and/or by dipping the plates in a cerium sulphate-ammonium molybdate solution followed by heating. Liquid column chromatography was performed using forced flow of the indicated solvent on Fluka silica gel 60 (40-63 $\mu \mathrm{m})$. The ${ }^{1} \mathrm{H}-\mathrm{NMR}$ spectra $(300 \mathrm{MHz})$ are expressed in ppm relative to $\mathrm{CHCl}_{3}(7.26 \mathrm{ppm})$ as internal reference, the coupling constants are reported in $\mathrm{Hz}$. The same is valid for ${ }^{13} \mathrm{C}-\mathrm{NMR}$ spectra $\left(75 \mathrm{MHz}\right.$, internal reference $\left.\mathrm{CDCl}_{3}: 77.0 \mathrm{ppm}\right)$. For ${ }^{19} \mathrm{~F}-\mathrm{NMR}\left(282 \mathrm{MHz}\right.$ ) spectra, $\mathrm{CCl}_{3} \mathrm{~F}$ $(\delta=0 \mathrm{ppm})$ was used as internal references. Optical rotations $[\alpha]_{\mathrm{D}}$ were recorded using a sodium lamp $(\lambda=589 \mathrm{~nm})$ at room temperature with a $10 \mathrm{~cm} / 1 \mathrm{~mL}$ cell. The solvent and the concentration are specified, e.g. $\mathrm{c}=1=10 \mathrm{mg} / \mathrm{mL}$. IR spectra were recorded in $\mathrm{CHCl}_{3}$ and are expressed in $\mathrm{cm}^{-1}$. LC-MS spectra were obtained on a Agilent 1100 LC MSD high-performance liquid chromatograph with a Waters Symmetry ${ }^{\circledR}$ C18 column $(3.9 \times 150 \mathrm{~mm}, 5 \mu \mathrm{m})$, using a defined gradient of water/iso-propanol $(80 / 20+0.1 \%$ TFA: solvent A) and acetonitrile/isopropanol (80/20 + 0.1\% TFA: solvent B) (flow rate $1 \mathrm{~mL} / \mathrm{min})$ : 0-1 $\mathrm{min}: 60 / 40(\mathrm{~A} / \mathrm{B}) ; 1-11 \mathrm{~min}$ : 60/40 to 0/100 (A/B); 11-20 min: 0/100 (A/B). The spectra were detected at 208, 234, 254 and $280 \mathrm{~nm}$. Preparative HPLC was performed on Waters HPLC apparatus with a Waters SunFire ${ }^{\mathrm{TM}}$ Prep C8 column $(10 \times 150 \mathrm{~mm}, 5 \mu \mathrm{m})$, using a gradient of water/iso-propanol $(80 / 20+0.1 \%$ TFA: solvent A) and acetonitrile/iso-propanol $(80 / 20+0.1 \%$ TFA: solvent B) (flow rate 5 
$\left.\mathrm{mL} \cdot \min ^{-1}\right)$ : $0-10 \mathrm{~min}: 100 / 0(\mathrm{~A} / \mathrm{B}) ; 10-50 \min : 100 / 0$ to $40 / 60(\mathrm{~A} / \mathrm{B}) ; 50-90 \mathrm{~min}: 40 / 60$ to $0 / 100$ (A/B); 90-100 min: 0/100 (A/B). The spectra were detected at a wavelength of 208 and $254 \mathrm{~nm}$. High-resolution mass spectroscopy (HRMS) was on a IonSpect Ultima; 2,5-dihydroxybenzoic acid (DHB) was used as matrix.

Compounds 2, 3 and $\mathbf{1 5}$ were obtained according to reported procedure ${ }^{1}$.

Pent-4-enyl 3,4-di- $\boldsymbol{O}$-benzyl-2-O-pivaloyl- $\boldsymbol{\beta}$-D-glucopyranoside (8). A solution of 7 (133 mg, $0.181 \mathrm{mmol})$ in piperidine/DMF $(0.75 \mathrm{~mL} / 3 \mathrm{~mL})$ was stirred for $30 \mathrm{~min}$ at room temperature. After solvent evaporation, the residue was purified by silica gel flash column chromatography (gradient eluent EtOAc/cyclohexane (1:7) to (1:3)) to afford 8 (92 $\mathrm{mg}, 99 \%)$ as a colorless oil. $\mathrm{R}_{\mathrm{f}}\left(\right.$ EtOAc/cyclohexane(1:4)) $=0.14 ;{ }^{1} \mathrm{H}-\mathrm{NMR}\left(300 \mathrm{MHz}, \mathrm{CDCl}_{3}\right): 7.37-7.24(\mathrm{~m}, 10 \mathrm{H}), 5.80$ (dddd, $J=17.0,10.4,6.6,6.6 \mathrm{~Hz}, 1 \mathrm{H}), 5.04$ (dd, $J=10.7,8.0 \mathrm{~Hz}, 1 \mathrm{H}), 5.02(\mathrm{dm}, J=17.0,1 \mathrm{H}$ ), $4.97(\mathrm{dm}, J=10.4 \mathrm{~Hz}, 1 \mathrm{H}), 4.82(\mathrm{~d}, J=11.0 \mathrm{~Hz}, 1 \mathrm{H}), 4.79(\mathrm{~d}, J=11.3 \mathrm{~Hz}, 1 \mathrm{H}), 4.71(\mathrm{~d}, J=11.3$ Hz, 1H), 4.64 (d, $J=11.0 \mathrm{~Hz}, 1 \mathrm{H}), 4.44(\mathrm{~d}, J=8.0 \mathrm{~Hz}, 1 \mathrm{H}), 3.89$ (ddd, $J=12.1,6.0,2.8 \mathrm{~Hz}, 1 \mathrm{H})$, 3.85 (ddd, $J=9.6,6.3,6.3 \mathrm{~Hz}, 1 \mathrm{H}), 3.68-3.64(\mathrm{~m}, 3 \mathrm{H}), 3.47$ (ddd, $J=9.6,6.9,6.9 \mathrm{~Hz}, 1 \mathrm{H}), 3.43$ (ddd, $J=9.1,4.4,2.8 \mathrm{~Hz}, 1 \mathrm{H}), 2.15-2.04$ (m, 2H), 1.99 (dd, $J=7.4,6.0 \mathrm{~Hz}, 1 \mathrm{H}), 1.67$ (quint., $J$ $=7.0 \mathrm{~Hz}, 2 \mathrm{H}), 1.21(\mathrm{~s}, 9 \mathrm{H}) ;{ }^{13} \mathrm{C}-\mathrm{NMR}\left(75 \mathrm{MHz}, \mathrm{CDCl}_{3}\right): 176.6,138.0,137.8,137.7,128.4$, $128.3,128.0,127.9,127.3,127.6,114.8,101.2,83.0,77.4,75.2,75.0,74.9,73.0,69.2,61.8$, 38.7, 29.9, 28.7, 27.1; $[\alpha]_{\mathrm{D}}\left(\mathrm{CHCl}_{3}, \mathrm{c}=1\right):-18.6^{\circ} ; \mathrm{IR}\left(\mathrm{CHCl}_{3}\right): 3619,3008,2976,1737,1522$, 1422, 1043, 928; HRMS(MALDI) calcd for $\mathrm{C}_{30} \mathrm{H}_{40} \mathrm{O}_{7} \mathrm{Na}$ 535.2666, found 535.2665.

Pent-4-enyl 6-O-acetyl-3,4-di- $O$-benzyl-2- $O$-pivaloyl- $\beta$-D-glucopyranoside (9). A solution of $8(25 \mathrm{mg}, 0.049 \mathrm{mmol})$ in pyridine $/ \mathrm{Ac}_{2} \mathrm{O}(1 \mathrm{~mL} / 0.5 \mathrm{~mL})$ was stirred for $1 \mathrm{~h}$ at room temperature. Solvents evaporation afforded $9(25 \mathrm{mg}, 93 \%)$ as a colorless oil. No further 
purification was required. Ret.time $(40-100)=9.6 \mathrm{~min} ; \mathrm{R}_{\mathrm{f}}($ EtOAc:hexane $(1: 4))=0.38 ;{ }^{1} \mathrm{H}-\mathrm{NMR}$ (300 MHz, $\left.\mathrm{CDCl}_{3}\right): 7.39-7.22(\mathrm{~m}, 10 \mathrm{H}), 5.79$ (dddd, $\left.J=17.0,10.4,6.6,6.6 \mathrm{~Hz}, 1 \mathrm{H}\right), 5.07$ (dd, $J$ $=9.0,8.7 \mathrm{~Hz}, 1 \mathrm{H}), 5.05-4.93(\mathrm{~m}, 2 \mathrm{H}), 4.82(\mathrm{~d}, J=11.0 \mathrm{~Hz}, 1 \mathrm{H}), 4.77(\mathrm{~d}, J=11.0 \mathrm{~Hz}, 1 \mathrm{H}), 4.72$ $(\mathrm{d}, J=11.0 \mathrm{~Hz}, 1 \mathrm{H}), 4.56(\mathrm{~d}, J=11.0 \mathrm{~Hz}, 1 \mathrm{H}), 4.40(\mathrm{~d}, J=7.7 \mathrm{~Hz}, 1 \mathrm{H}), 4.38(\mathrm{dd}, J=12.1,2.2$ $\mathrm{Hz}, 1 \mathrm{H}), 4.23(\mathrm{dd}, J=12.1,5.0 \mathrm{~Hz}, 1 \mathrm{H}), 3.84(\mathrm{ddd}, J=9.6,6.6,6.6 \mathrm{~Hz}, 1 \mathrm{H}), 3.74$ (dd, $J=9.0$, $8.5 \mathrm{~Hz}, 1 \mathrm{H}), 3.65$ (dd, 1H, $J=9.6,8.5 \mathrm{~Hz}), 3.55$ (ddd, $J=9.6,5.0,2.2 \mathrm{~Hz}, 1 \mathrm{H}), 3.46$ (ddd, $J=9.6$, 6.6, $6.6 \mathrm{~Hz}, 1 \mathrm{H}), 2.14-2.03(\mathrm{~m}, 2 \mathrm{H}), 2.05(\mathrm{~s}, 3 \mathrm{H}), 1.72-1.61(\mathrm{~m}, 2 \mathrm{H}), 1.21(\mathrm{~s}, 9 \mathrm{H}){ }^{13} \mathrm{C}-\mathrm{NMR}$ $\left(75 \mathrm{MHz}, \mathrm{CDCl}_{3}\right): 176.6,170.7,137.9,137.9,137.5,128.4,128.3,128.0,127.9,127.6,127.3$, $114.8,101.2,83.2,77.3,74.9,72.9,69.0,62.8,38.7,29.9,28.7,27.1,20.8 ;[\alpha]_{\mathrm{D}}\left(\mathrm{CHCl}_{3}, \mathrm{c}=1\right)$ : $-4.9^{\circ} ; \mathrm{IR}\left(\mathrm{CHCl}_{3}\right): 3008,2974,1737,1479,1454,1365,1248,1144,1087,1039,917,879$; HRMS(MALDI) calcd for $\mathrm{C}_{32} \mathrm{H}_{42} \mathrm{O}_{8} \mathrm{Na}$ 577.2772, found 577.2765.

\section{Pent-4-enyl 2-O-acetyl-3,4-di- $O$-benzyl-6-O-(fluorenylmethoxycarbonyl)- $\alpha$-D-} mannopyranosyl-(1 $\rightarrow 6)$-3,4-di- $O$-benzyl-2- $O$-pivaloyl- $\beta$-D-glucopyranoside $\quad(10)$. General procedure for glycosylation in solution using $8(102 \mathrm{mg}, 0.20 \mathrm{mmol}), 3(211 \mathrm{mg}, 0.26 \mathrm{mmol})$, molecular sieves $(200 \mathrm{mg})$ and TMSOTf $(47 \mu \mathrm{L}, 0.26 \mathrm{mmol})$ in $\mathrm{CH}_{2} \mathrm{Cl}_{2}(4 \mathrm{~mL})$ at $-30{ }^{\circ} \mathrm{C}$ for 15 min. The reaction was quenched with pyridine $(100 \mu \mathrm{L})$. Silica gel flash column chromatography (gradient eluent EtOAc/cyclohexane (1:9) to (1:4)) afforded $\mathbf{1 0}(198 \mathrm{mg}, 89 \%)$ as a white foam. $\mathrm{R}_{\mathrm{f}}($ EtOAc/cyclohexane $(1: 4))=0.31 ;{ }^{1} \mathrm{H}-\mathrm{NMR}\left(300 \mathrm{MHz}, \mathrm{CDCl}_{3}\right): 7.78(\mathrm{~d}, J=7.5 \mathrm{~Hz}, 2 \mathrm{H}), 7.67-$ 7.61 (m, 2H), $7.45-7.20$ (m, 24H), 5.79 (dddd, $J=17.1,10.3,6.5,6.5 \mathrm{~Hz}, 1 \mathrm{H}), 5.47$ (dd, $J=3.1$, $1.9 \mathrm{~Hz}, 1 \mathrm{H}), 5.10$ (dd, $J=9.3,8.1 \mathrm{~Hz}, 1 \mathrm{H}), 5.06-4.92(\mathrm{~m}, 4 \mathrm{H}), 4.85-4.70(\mathrm{~m}, 4 \mathrm{H}), 4.58$ (d, $J=$ $11.2 \mathrm{~Hz}, 2 \mathrm{H}), 4.50$ (d, $J=11.2 \mathrm{~Hz}, 1 \mathrm{H}), 4.45-4.34$ (m, 5H), 4.27 (t, $J=7.5 \mathrm{~Hz}, 1 \mathrm{H}), 4.01$ (dd, $J$ $=8.4,3.1 \mathrm{~Hz}, 1 \mathrm{H}), 3.92-3.70(\mathrm{~m}, 6 \mathrm{H}), 3.61-3.38(\mathrm{~m}, 3 \mathrm{H}), 2.18(\mathrm{~s}, 3 \mathrm{H}), 2.13-2.02(\mathrm{~m}, 2 \mathrm{H})$, 1.72 - 1.55 (m, 2H), 1.24 (s, 9H); ${ }^{13} \mathrm{C}-\mathrm{NMR}\left(75 \mathrm{MHz}, \mathrm{CDCl}_{3}\right)$ : 176.5, 170.0, 154.9, 143.4, 143.2, 
$141.2,138.1,138.0,137.9,137.7,137.5,128.4,128.3,128.3,128.2,128.2,127.8,127.7,127.6$, $127.5,127.4,127.1,127.1,125.1,125.1,120.0,114.7,101.0,97.7,83.4,77.7,77.6,75.2,75.0$, $74.9,74.2,73.8,73.1,71.6,69.9,69.8,68.9,68.2,66.6,66.2,46.8,38.9,30.2,28.9,27.3,21.2$; $[\alpha]_{\mathrm{D}}\left(\mathrm{CHCl}_{3}, \mathrm{c}=1\right): 6.7^{\circ} ; \mathrm{IR}\left(\mathrm{CHCl}_{3}\right): 3068,3008,2988,2874,1741,1640,1605,1497,1478$, 1452, 1397, 1370, 1260, 1092, 974, 916; HRMS(MALDI) calcd for $\mathrm{C}_{67} \mathrm{H}_{74} \mathrm{O}_{15} \mathrm{Na} 1141.4920$, found 1141.490 .

Pent-4-enyl 2-O-acetyl-3,4-di- $O$-benzyl- $\alpha$-D-mannopyranosyl-(1 $\rightarrow 6)$-3,4-di- $O$-benzyl-2- $O$ pivaloyl- $\beta$-D-glucopyranoside (11). A solution of 10 (176 $\mathrm{mg}, 0.157 \mathrm{mmol})$ in piperidine/DMF $(0.6 \mathrm{~mL} / 2.4 \mathrm{~mL})$ was stirred for $30 \mathrm{~min}$ at room temperature. The solvents were evaporated and the residue was purified by silica gel flash column chromatography (gradient eluent EtOAc/cyclohexane (1:4) to (1:2)) to afford 11 (142 $\mathrm{mg}$, quantitative) as a colorless oil. $\mathrm{R}_{\mathrm{f}}\left(\right.$ EtOAc/cyclohexane(1:1.5) $=0.63 ;{ }^{1} \mathrm{H}-\mathrm{NMR}\left(300 \mathrm{MHz}, \mathrm{CDCl}_{3}\right): 7.35-7.17(\mathrm{~m}, 20 \mathrm{H}), 5.76$ (dddd, $J=17.1,10.3,6.5,6.5 \mathrm{~Hz}, 1 \mathrm{H}), 5.41(\mathrm{dd}, J=3.4,1.9 \mathrm{~Hz}, 1 \mathrm{H}), 5.05(\mathrm{dd}, J=9.3,8.1 \mathrm{~Hz}$, 1H), $5.02-4.87(\mathrm{~m}, 3 \mathrm{H}), 4.84(\mathrm{~d}, J=1.9 \mathrm{~Hz}, 1 \mathrm{H}), 4.80(\mathrm{~d}, J=11.2 \mathrm{~Hz}, 1 \mathrm{H}), 4.73(\mathrm{~d}, J=11.2 \mathrm{~Hz}$, 1H), $4.72(\mathrm{bs}, 2 \mathrm{H}), 4.59(\mathrm{~d}, J=11.2 \mathrm{~Hz}, 1 \mathrm{H}), 4.53(\mathrm{~d}, J=11.5 \mathrm{~Hz}, 1 \mathrm{H}), 4.47(\mathrm{~d}, J=11.2 \mathrm{~Hz}$, $1 \mathrm{H}), 4.36(\mathrm{~d}, J=8.1 \mathrm{~Hz}, 1 \mathrm{H}), 3.93(\mathrm{dd}, J=9.0,3.1 \mathrm{~Hz}, 1 \mathrm{H}), 3.84-3.36(\mathrm{~m}, 11 \mathrm{H}), 2.14(\mathrm{~s}, 3 \mathrm{H})$, $2.10-1.98(\mathrm{~m}, 2 \mathrm{H}), 1.70(\mathrm{dd}, J=7.8,5.0 \mathrm{~Hz}, 1 \mathrm{H}), 1.66-1.52(\mathrm{~m}, 2 \mathrm{H}), 1.20(\mathrm{~s}, 9 \mathrm{H}) ;{ }^{13} \mathrm{C}-\mathrm{NMR}$ (75 MHz, $\left.\mathrm{CDCl}_{3}\right): 176.5,170.0,138.3,138.0,137.8,137.7,137.6,128.3,128.2,128.0,127.3$, $127.5,127.3,114.7,101.0,97.8,83.4,77.7,75.1,75.0,74.9,74.2,73.9,73.1,71.9,71.5,69.0$, 68.3, 66.0, 62.0, 38.9, 30.1, 28.9, 27.3, 21.1; $[\alpha]_{\mathrm{D}}\left(\mathrm{CHCl}_{3}, \mathrm{c}=1\right): 10.2^{\circ}$; IR $\left(\mathrm{CHCl}_{3}\right): 3606,3068$, 3008, 2933, 2875, 1738, 1497, 1479, 1455, 1370, 1278, 1089, 911, 818; HRMS(MALDI) calcd for $\mathrm{C}_{52} \mathrm{H}_{64} \mathrm{O}_{13} \mathrm{Na} 919.4239$, found 919.4222 . 
Pent-4-enyl 2,6-di- $O$-acetyl-3,4-di- $O$-benzyl- $\alpha$-D-mannopyranosyl-(1 $\rightarrow$ 6)-3,4-di- $O$-benzyl2-O-pivaloyl- $\boldsymbol{\beta}$-D-glucopyranoside (12). A solution of 11 (41 mg, $46 \mu \mathrm{mol})$ in pyridine/ $\mathrm{Ac}_{2} \mathrm{O}$ (1 $\mathrm{mL} / 0.5 \mathrm{~mL}$ ) was stirred for $60 \mathrm{~min}$ at room temperature. The solvents were evaporated and the residue was purified by silica gel flash column chromatography (gradient eluent EtOAc/cyclohexane (1:4) to (1:3)) to afford $11(37 \mathrm{mg}, 86 \%)$ as a colorless oil. Ret.time $(40-100)=11.3 \mathrm{~min} ; \mathrm{R}_{\mathrm{f}}($ EtOAc/cyclohexane $(1: 3))=0.32 ;{ }^{1} \mathrm{H}-\mathrm{NMR}\left(300 \mathrm{MHz}, \mathrm{CDCl}_{3}\right): 7.37$ - 7.16 (m, 20H), 5.76 (dddd, $J=17.1,10.3,6.5,6.5 \mathrm{~Hz}, 1 \mathrm{H}), 5.42$ (dd, $J=3.1,1.9 \mathrm{~Hz}, 1 \mathrm{H}), 5.05$ (dd, $J$ $=9.0,8.1 \mathrm{~Hz}, 1 \mathrm{H}), 5.02-4.85(\mathrm{~m}, 4 \mathrm{H}), 4.81(\mathrm{~d}, J=10.9 \mathrm{~Hz}, 1 \mathrm{H}), 4.77-4.67(\mathrm{~m}, 3 \mathrm{H}), 4.53(\mathrm{~d}, J$

$=10.9 \mathrm{~Hz}, 1 \mathrm{H}), 4.53(\mathrm{~d}, J=10.6 \mathrm{~Hz}, 1 \mathrm{H}), 4.48(\mathrm{~d}, J=11.2 \mathrm{~Hz}, 1 \mathrm{H}), 4.37(\mathrm{~d}, J=7.8 \mathrm{~Hz}, 1 \mathrm{H})$, $4.29-4.17(\mathrm{~m}, 2 \mathrm{H}), 3.95(\mathrm{dd}, J=9.0,3.1 \mathrm{~Hz}, 1 \mathrm{H}), 3.86-3.64(\mathrm{~m}, 6 \mathrm{H}), 3.58-3.34(\mathrm{~m}, 3 \mathrm{H}), 2.15$ (s, 3H), $2.09-1.94(\mathrm{~m}, 5 \mathrm{H}), 1.66-1.54(\mathrm{~m}, 2 \mathrm{H}), 1.21(\mathrm{~s}, 9 \mathrm{H}) ;{ }^{13} \mathrm{C}-\mathrm{NMR}\left(75 \mathrm{MHz}, \mathrm{CDCl}_{3}\right)$ : $176.5,170.5,169.9,138.0,138.0,137.8,137.6,137.5,128.3,128.3,128.2,128.1,127.8,127.6$, $127.5,127.4,114.7,101.0,97.7,83.4,77.7,77.6,75.1,75.0,74.9,74.1,73.8,73.1,71.6,69.8$, 68.9, 68.1, 66.2, 62.3, 38.9, 30.1, 28.8, 27.2, 21.4, 20.9; $[\alpha]_{\mathrm{D}}\left(\mathrm{CHCl}_{3}, \mathrm{c}=1\right): 10.0^{\circ} ; \mathrm{IR}\left(\mathrm{CHCl}_{3}\right)$ : 3066, 3008, 2969, 2874, 1738, 1640, 1497, 1479, 1454, 1397, 1369, 1248, 1091, 979, 915; HRMS(MALDI) calcd for $\mathrm{C}_{54} \mathrm{H}_{66} \mathrm{O}_{14} \mathrm{Na} 961.4345$, found 961.4351 .

Pent-4-enyl 3,4-di- $O$-benzyl-6- $O$-(fluorenylmethoxycarbonyl)-2- $O$-pivaloyl- $\beta$-Dglucopyranosyl-(1 $\rightarrow 6)-2-O$-acetyl-3,4-di-O-benzyl- $\alpha$-D-mannopyranosyl-(1 $\rightarrow 6)-3,4-$ di- $O$ benzyl-2-O-pivaloyl- $\beta$-D-glucopyranoside (13). General procedure for glycosylation in solution using 11 (89 mg, $0.1 \mathrm{mmol}), 2$ (111 mg, $0.13 \mathrm{mmol})$, molecular sieves (200 mg) and TMSOTf $(23 \mu \mathrm{L}, 0.13 \mathrm{mmol})$ in $\mathrm{CH}_{2} \mathrm{Cl}_{2}(2 \mathrm{~mL})$ at $-30{ }^{\circ} \mathrm{C}$ for $15 \mathrm{~min}$. The reaction was quenched with pyridine $(100 \mu \mathrm{L})$. Silica gel flash column chromatography (gradient eluent EtOAc/cyclohexane (1:9) to (1:4)) afforded $13(131 \mathrm{mg}, 85 \%)$ as a white foam. $\mathrm{R}_{\mathrm{f}}($ EtOAc/cyclohexane $(1: 4))=0.26$; 
${ }^{1} \mathrm{H}-\mathrm{NMR}\left(300 \mathrm{MHz}, \mathrm{CDCl}_{3}\right)$ : $7.75(\mathrm{~d}, J=7.5 \mathrm{~Hz}, 2 \mathrm{H}), 7.63$ - $7.55(\mathrm{~m}, 2 \mathrm{H}), 7.42$ - $7.14(\mathrm{~m}, 34 \mathrm{H})$, 5.77 (dddd, $J=17.1,10.3 \mathrm{~Hz}, 6.5,6.5,1 \mathrm{H}), 5.40(\mathrm{dd}, J=3.1,1.9 \mathrm{~Hz}, 1 \mathrm{H}), 5.18(\mathrm{dd}, J=8.7,7.5$ $\mathrm{Hz}, 1 \mathrm{H}), 5.06(\mathrm{dd}, J=9.0,8.1 \mathrm{~Hz}, 1 \mathrm{H}), 5.03-4.91(\mathrm{~m}, 2 \mathrm{H}), 4.90-4.85(\mathrm{~m}, 2 \mathrm{H}), 4.82(\mathrm{~d}, J=10.9$ $\mathrm{Hz}, 1 \mathrm{H}), 4.79-4.65(\mathrm{~m}, 5 \mathrm{H}), 4.61-4.28(\mathrm{~m}, 11 \mathrm{H}), 4.21(\mathrm{t}, J=7.5 \mathrm{~Hz}, 1 \mathrm{H}), 4.08(\mathrm{bd}, 1 \mathrm{H}, J=$ $11.0 \mathrm{~Hz}), 3.92(\mathrm{dd}, J=9.0,3.1 \mathrm{~Hz}, 1 \mathrm{H}), 3.88-3.53(\mathrm{~m}, 11 \mathrm{H}), 3.48-3.34(\mathrm{~m}, 2 \mathrm{H}), 2.15(\mathrm{~s}, 3 \mathrm{H})$, 2.10 - $2.00(\mathrm{~m}, 2 \mathrm{H}), 1.66$ - $1.54(\mathrm{~m}, 2 \mathrm{H}), 1.21(\mathrm{~s}, 9 \mathrm{H}), 1.20(\mathrm{~s}, 9 \mathrm{H}) ;{ }^{13} \mathrm{C}-\mathrm{NMR}\left(75 \mathrm{MHz}, \mathrm{CDCl}_{3}\right)$ : $176.5,176.3,170.1,154.9,143.3,143.2,141.2,138.4,138.1,138.0,137.9,137.8,137.5,128.4$, $128.3,128.3,128.2,127.9,127.8,127.6,127.5,127.3,127.2,127.1,125.2,125.1,120.0,114.7$, $101.0,101.0,97.6,83.5,83.2,77.6,77.4,77.3,75.0,74.9,74.8,74.3,74.2,73.1,72.9,71.3,70.7$, 70.0, 68.8, 68.2, 68.0, 66.7, 65.6, 46.8, 38.9, 30.2, 28.9, 27.4, 27.3, 21.2; $[\alpha]_{\mathrm{D}}\left(\mathrm{CHCl}_{3}, \mathrm{c}=1\right): 0.6^{\circ}$; IR ( $\left.\mathrm{CHCl}_{3}\right): 3066,3032,2959,2874,1739,1605,1497,1479,1453,1397,1364,1263,1090$, 913; HRMS(MALDI) calcd for $\mathrm{C}_{92} \mathrm{H}_{104} \mathrm{O}_{21} \mathrm{Na} 1567.6968$, found 1567.6967 .

Pent-4-enyl 3,4-di- $O$-benzyl-2,6-di- $O$-pivaloyl- $\beta$-D-glucopyranosyl-(1 $\rightarrow$ 6)-3,4-di- $O$-benzyl2-O-pivaloyl- $\beta$-D-glucopyranosyl-(1 $\rightarrow 6$ )-3,4-di- $O$-benzyl-2- $O$-pivaloyl- $\beta$-D-glucopyranoside

(17). To a stirred solution of $\mathbf{2 0}(43 \mathrm{mg}, 31.5 \mu \mathrm{mol})$ in pyridine $(1 \mathrm{~mL})$ was added $\mathrm{PivCl}(15.5$ $\mu \mathrm{L}, 0.126 \mathrm{mmol})$ at room temperature. After $135 \mathrm{~min}$, the solvents were evaporated. The residue was purified by silica gel flash column chromatography (gradient eluent EtOAc/cyclohexane (1:9) to (1:7)) to afford $17(41 \mathrm{mg}, 91 \%)$ as a white foam. Ret.time $(40-100)=13.2 \mathrm{~min}$; $\mathrm{R}_{\mathrm{f}}($ EtOAc/cyclohexane $(1: 7))=0.16 ;{ }^{1} \mathrm{H}-\mathrm{NMR}\left(300 \mathrm{MHz}, \mathrm{CDCl}_{3}\right): 7.36-7.20(\mathrm{~m}, 30 \mathrm{H}), 5.81$ (dddd, $J=17.0,10.2,6.4,6.4 \mathrm{~Hz}, 1 \mathrm{H}), 5.11-4.95(\mathrm{~m}, 5 \mathrm{H}), 4.82-4.66(\mathrm{~m}, 9 \mathrm{H}), 4.65-4.55(\mathrm{~m}$, $3 \mathrm{H}), 4.52(\mathrm{~d}, J=10.7 \mathrm{~Hz}, 1 \mathrm{H}), 4.47(\mathrm{~d}, J=8.0 \mathrm{~Hz}, 1 \mathrm{H}), 4.41(\mathrm{dd}, J=12.1,1.9 \mathrm{~Hz}, 1 \mathrm{H}), 4.36(\mathrm{~d}$, $J=8.0 \mathrm{~Hz}, 1 \mathrm{H}), 4.18(\mathrm{dd}, J=12.1,4.9 \mathrm{~Hz}, 1 \mathrm{H}), 4.02(\mathrm{dd}, J=11.5,1.7 \mathrm{~Hz}, 1 \mathrm{H}), 3.97(\mathrm{bd}, J=$ $11.3 \mathrm{~Hz}, 1 \mathrm{H}), 3.86(\mathrm{ddd}, J=9.3,6.3,6.3 \mathrm{~Hz}, 1 \mathrm{H}), 3.79-3.40(\mathrm{~m}, 12 \mathrm{H}), 2.15-2.05(\mathrm{~m}, 2 \mathrm{H})$, 
$1.72-1.60(\mathrm{~m}, 2 \mathrm{H}), 1.20(\mathrm{~s}, 18 \mathrm{H}), 1.20(\mathrm{~s}, 9 \mathrm{H}), 1.20(\mathrm{~s}, 9 \mathrm{H}) ;{ }^{13} \mathrm{C}-\mathrm{NMR}\left(75 \mathrm{MHz}, \mathrm{CDCl}_{3}\right): 178.0$, $176.6,176.5,138.0,137.9,137.9,137.8,137.8,137.7,137.6,128.4,128.4,128.3,128.2,127.9$, $127.8,127.6,127.5,127.3,127.2,127.2,114.8,100.9,100.7,100.6,83.1,83.0,78.2,78.0,77.6$, $75.4,75.0,74.9,74.9,74.7,73.2,72.8,72.7,68.8,67.7,67.2,62.6,38.8,38.7,30.0,28.7,27.2$, 27.1, 27.1, 27.1; $[\alpha]_{\mathrm{D}}\left(\mathrm{CHCl}_{3}, \mathrm{c}=1\right):-19.8^{\circ} ; \mathrm{IR}\left(\mathrm{CHCl}_{3}\right): 3066,3008,2971,2934,2873,1736$, $1602,1497,1479,1455,1397,1363,1279,1141,1089,1037,941,913,885$; HRMS(MALDI) calcd for $\mathrm{C}_{85} \mathrm{H}_{108} \mathrm{O}_{20} \mathrm{Na}$ 1471.7326, found 1471.7355 .

Pent-4-enyl 3,4-di- $O$-benzyl-2,6-di- $O$-pivaloyl- $\beta$-D-glucopyranosyl-(1 $\rightarrow 6)$-3,4-di- $O$-benzyl2-O-pivaloyl- $\beta$-D-glucopyranoside (18). To a stirred solution of $22(36 \mathrm{mg}, 38 \mu \mathrm{mol})$ in pyridine $(1 \mathrm{~mL})$ was added $\mathrm{PivCl}(19 \mu \mathrm{L}, 154 \mu \mathrm{mol})$ at room temperature. After $90 \mathrm{~min}$, the solvents were evaporated. The residue was purified by silica gel flash column chromatography (eluent EtOAc/cyclohexane (1:4)) to afford $\mathbf{1 8}(36 \mathrm{mg}, 91 \%)$ as a white foam. Ret.time $(40-100)=12.4 \mathrm{~min} ; \mathrm{R}_{\mathrm{f}}($ EtOAc/cyclohexane $(1: 4))=0.43 ;{ }^{1} \mathrm{H}-\mathrm{NMR}\left(300 \mathrm{MHz}, \mathrm{CDCl}_{3}\right): 7.38-7.21$ (m, 20H), 5.80 (dddd, $J=17.0,10.2,6.6,6.6 \mathrm{~Hz}, 1 \mathrm{H}), 5.10-4.94$ (m, 4H), 4.82 (d, $J=11.2 \mathrm{~Hz}$, 1H), $4.77(\mathrm{~d}, J=11.2 \mathrm{~Hz}, 1 \mathrm{H}), 4.76(\mathrm{~d}, J=11.2 \mathrm{~Hz}, 1 \mathrm{H}), 4.74(\mathrm{~d}, J=11.2 \mathrm{~Hz}, 1 \mathrm{H}), 4.69$ (d, $J=$ $11.2 \mathrm{~Hz}, 1 \mathrm{H}), 4.68(\mathrm{~d}, J=11.2 \mathrm{~Hz}, 1 \mathrm{H}), 4.59$ (d, $J=8.0 \mathrm{~Hz}, 1 \mathrm{H}), 4.56(\mathrm{~d}, J=11.2 \mathrm{~Hz}, 1 \mathrm{H}), 4.55$ $(\mathrm{d}, J=11.2 \mathrm{~Hz}, 1 \mathrm{H}), 4.45(\mathrm{dd}, J=12.1,1.8 \mathrm{~Hz}, 1 \mathrm{H}), 4.34(\mathrm{~d}, J=8.0 \mathrm{~Hz}, 1 \mathrm{H}), 4.215(\mathrm{dd}, J=$ 12.1, $4.5 \mathrm{~Hz}, 1 \mathrm{H}), 4.01$ (dd, $J=11.3,1.4 \mathrm{~Hz}, 1 \mathrm{H}), 3.87$ (ddd, $J=9.6,6.3,6.3 \mathrm{~Hz}, 1 \mathrm{H}), 3.75-3.36$ (m, 8H), $2.20-2.00(\mathrm{~m}, 2 \mathrm{H}), 1.72-1.58(\mathrm{~m}, 2 \mathrm{H}), 1.21(\mathrm{~s}, 9 \mathrm{H}), 1.20(\mathrm{~s}, 9 \mathrm{H}), 1.20(\mathrm{~s}, 9 \mathrm{H}) ;{ }^{13} \mathrm{C}-$ NMR (75 MHz, $\left.\mathrm{CDCl}_{3}\right)$ : 178.0, 176.5, 137.9, 137.8, 137.6, 137.4, 128.4, 128.4, 128.3, 128.3, $127.9,127.6,127.5,127.4,127.3,114.8,100.8,100.7,83.1,83.1,78.1,77.7,75.4,75.0,75.0$, 74.9, 74.9, 73.3, 72.9, 72.7, 68.9, 67.3, 62.6, 38.8, 38.7, 30.0, 28.7, 27.1, 27.1; $[\alpha]_{\mathrm{D}}\left(\mathrm{CHCl}_{3}, \mathrm{c}=1\right)$ : $-13.0^{\circ}$; IR $\left(\mathrm{CHCl}_{3}\right): 3067,3008,2906,2873,1736,1640,1497,1479,1455,1397,1363,1280$, 
1262, 1142, 1090, 1037, 1016, 913; HRMS(MALDI) calcd for $\mathrm{C}_{60} \mathrm{H}_{78} \mathrm{O}_{14} \mathrm{Na} 1045.5284$, found 1045.5282.

Pent-4-enyl 3,4-di- $\boldsymbol{O}$-benzyl-2,6-di- $\boldsymbol{O}$-pivaloyl- $\boldsymbol{\beta}$-D-glucopyranoside (19). To a stirred solution of 8 (48 $\mathrm{mg}, 94 \mu \mathrm{mol})$ in pyridine $(1.5 \mathrm{~mL})$ was added PivCl $(47 \mu \mathrm{L}, 0.38 \mathrm{mmol})$ at room temperature. After $2 \mathrm{~h}$, the solvents were evaporated. Residual pyridine was removed by coevaporation with toluene $(2 \times 3 \mathrm{~mL})$. The residue was purified by silica gel flash column chromatography (eluent EtOAc/cyclohexane (1:5)) to afford 19 (54 mg, 96\%) as a white solid. Ret.time $(40-100)=10.9 \mathrm{~min} ; \mathrm{R}_{\mathrm{f}}($ EtOAc/cyclohexane $(1: 4))=0.57 ; \mathrm{mp}=50-52^{\circ} \mathrm{C} ;{ }^{1} \mathrm{H}-\mathrm{NMR}(300$ $\left.\mathrm{MHz}, \mathrm{CDCl}_{3}\right): 7.37-7.23(\mathrm{~m}, 10 \mathrm{H}), 5.78(\mathrm{dddd}, J=17.1,10.3,6.6,6.6 \mathrm{~Hz}, 1 \mathrm{H}), 5.08$ - $4.92(\mathrm{~m}$, 3H), $4.83(\mathrm{~d}, J=10.9 \mathrm{~Hz}, 1 \mathrm{H}), 4.77$ (d, $J=10.9 \mathrm{~Hz}, 1 \mathrm{H}), 4.71(\mathrm{~d}, J=10.9 \mathrm{~Hz}, 1 \mathrm{H}), 4.57$ (d, $J=$ $10.9 \mathrm{~Hz}, 1 \mathrm{H}), 4.46(\mathrm{dd}, J=12.1,2.2 \mathrm{~Hz}, 1 \mathrm{H}), 4.40(\mathrm{~d}, J=8.1 \mathrm{~Hz}, 1 \mathrm{H}), 4.18(\mathrm{dd}, J=12.1,5.0 \mathrm{~Hz}$, 1H), 3.81 (ddd, $J=9.6,6.3,6.3 \mathrm{~Hz}, 1 \mathrm{H}), 3.73(\mathrm{dd}, J=8.8,8.8 \mathrm{~Hz}, 1 \mathrm{H}), 3.66-3.52(\mathrm{~m}, 2 \mathrm{H}), 3.45$ (ddd, $J=9.6,6.3,6.3 \mathrm{~Hz}, 1 \mathrm{H}$ ), $2.14-2.02(\mathrm{~m}, 2 \mathrm{H}), 1.66$ (quint., $J=7.1 \mathrm{~Hz}, 2 \mathrm{H}$ ), $1.22(\mathrm{~s}, 9 \mathrm{H})$, 1.21 (s, 9H); ${ }^{13} \mathrm{C}-\mathrm{NMR}\left(75 \mathrm{MHz}, \mathrm{CDCl}_{3}\right): 177.9,176.5,137.8,137.8,137.5,128.4,128.3,127.9$, $127.6,127.4,114.8,101.0,83.2,77.9,75.2,73.2,73.1,68.9,62.8,38.9,38.9,30.1,28.8,27.3$, 27.2; $[\alpha]_{\mathrm{D}}\left(\mathrm{CHCl}_{3}, \mathrm{c}=1\right):-10.8^{\circ} ; \mathrm{IR}\left(\mathrm{CHCl}_{3}\right): 3067,3008,2975,2873,1731,1640,1497,1479$, $1455,1397,1364,1282,1149,1089,1037,1014,916$; HRMS(MALDI) calcd for $\mathrm{C}_{35} \mathrm{H}_{48} \mathrm{O}_{8} \mathrm{Na}$ 619.3242 , found 619.3246 .

Pent-4-en-1-yl 3,4-di- $O$-benzyl-2- $O$-pivaloyl- $\beta$-D-glucopyranosyl-(1 $\rightarrow 6)-3,4-d i-O$-benzyl-2$O$-pivaloyl- $\beta$-D-glucopyranosyl-( $1 \rightarrow 6)-3,4-$ di- $O$-benzyl-2- $O$-pivaloyl- $\beta$-D-glucopyranoside

(20). To a stirred solution of $\mathbf{2 3}(81 \mathrm{mg}, 51 \mu \mathrm{mol})$ in DMF $(0.8 \mathrm{~mL})$ was added piperidine $(0.2$ $\mathrm{mL}$ ) at room temperature. The solution was stirred for $30 \mathrm{~min}$ at room temperature and the solvents were evaporated. Residual piperidine was coevaporated with toluene $(2 \times 5 \mathrm{~mL})$. The 
residue was purified by silica gel flash column chromatography (gradient eluent EtOAc/cyclohexane (1:9) to (1:4)) to afford $\mathbf{2 0}(66 \mathrm{mg}, 95 \%)$ as a white foam. Ret.time $(40 / 100)=12.6 \mathrm{~min} ; \mathrm{R}_{\mathrm{f}}($ EtOAc/cyclohexane $(1: 4))=0.33 ;{ }^{1} \mathrm{H}-\mathrm{NMR}\left(300 \mathrm{MHz}, \mathrm{CDCl}_{3}\right)$ : $7.37-7.23(\mathrm{~m}, 30 \mathrm{H}), 5.81(\mathrm{dddd}, J=17.1,10.3,6.6,6.6 \mathrm{~Hz}, 1 \mathrm{H}), 5.10-4.94(\mathrm{~m}, 5 \mathrm{H}), 4.81(\mathrm{~d}, J$ $=11.0 \mathrm{~Hz}, 1 \mathrm{H}), 4.79(\mathrm{~d}, J=11.0 \mathrm{~Hz}, 2 \mathrm{H}), 4.76(\mathrm{~d}, J=11.0 \mathrm{~Hz}, 1 \mathrm{H}), 4.75(\mathrm{~d}, J=11.0 \mathrm{~Hz}, 2 \mathrm{H})$, $4.70(\mathrm{~d}, J=11.0 \mathrm{~Hz}, 1 \mathrm{H}), 4.68(\mathrm{~d}, J=11.0 \mathrm{~Hz}, 1 \mathrm{H}), 4.67(\mathrm{~d}, J=11.0 \mathrm{~Hz}, 1 \mathrm{H}), 4.63(\mathrm{~d}, J=11.0$ $\mathrm{Hz}, 1 \mathrm{H}), 4.61(\mathrm{~d}, J=11.0 \mathrm{~Hz}, 1 \mathrm{H}), 4.60(\mathrm{~d}, J=11.0 \mathrm{~Hz}, 1 \mathrm{H}), 4.53(\mathrm{~d}, J=8.1 \mathrm{~Hz}, 1 \mathrm{H}), 4.46(\mathrm{~d}, J$ $=7.8 \mathrm{~Hz}, 1 \mathrm{H}), 4.38(\mathrm{~d}, J=7.8 \mathrm{~Hz}, 1 \mathrm{H}), 3.98-3.32(\mathrm{~m}, 17 \mathrm{H}), 2.47(\mathrm{dd}, J=6.5,6.5 \mathrm{~Hz}, 1 \mathrm{H}), 2.15$ - $2.05(\mathrm{~m}, 2 \mathrm{H}), 1.71-1.61$ (quint., $J=7.2 \mathrm{~Hz}, 2 \mathrm{H}), 1.21(\mathrm{~s}, 9 \mathrm{H}), 1.20(\mathrm{~s}, 9 \mathrm{H}), 1.18(\mathrm{~s}, 9 \mathrm{H}) ;{ }^{13} \mathrm{C}-$ NMR (75 MHz, $\left.\mathrm{CDCl}_{3}\right)$ : 176.6, 176.4, 176.4, 143.3, 143.1, 141.1, 141.1, 138.0, 138.0, 137.9, $137.8,137.6,128.3,128.2,128.0,127.9,127.8,127.5,127.3,127.2,114.8,101.8,101.1,110.8$, $83.2,83.1,83.0,78.0,77.7,77.5,75.7,75.3,74.9,74.8,74.7,73.1,72.9,69.3,69.0,68.2,61.9$, 38.9, 30.2, 28.9, 27.3; $[\alpha]_{\mathrm{D}}\left(\mathrm{CHCl}_{3}, \mathrm{c}=0.5\right):-13.2^{\circ}$; IR $\left(\mathrm{CHCl}_{3}\right): 3474,3065,3008,2971,2875$, 1737, 1602, 1520, 1497, 1479, 1454, 1397, 1363, 1277, 1262, 1139, 1086; HRMS(MALDI) calcd for $\mathrm{C}_{80} \mathrm{H}_{100} \mathrm{O}_{19} \mathrm{Na}$ 1387.6751, found 1387.6778.

\section{Pent-4-enyl 3,4-di- $O$-benzyl-2- $O$-pivaloyl- $\beta$-D-glucopyranosyl-(1 $\rightarrow$ 6)-3,4-di- $O$-benzyl-2-O-}

pivaloyl- $\beta$-D-glucopyranoside (22). General procedure for glycosylation in solution using 8 (92 mg, $0.179 \mathrm{mmol}), 2(200 \mathrm{mg}, 0.233 \mathrm{mmol})$ and TMSOTf $(42 \mu \mathrm{L}, 0.233 \mathrm{mmol})$ in $\mathrm{CH}_{2} \mathrm{Cl}_{2}(3.6$ $\mathrm{mL})$ between $-30{ }^{\circ} \mathrm{C}$ and $-20^{\circ} \mathrm{C}$ for $30 \mathrm{~min}$. The reaction was quenched with pyridine $(150 \mu \mathrm{L})$. Solvents evaporation afforded crude 21, which was stirred in piperidine/DMF $(0.75 \mathrm{~mL} / 3 \mathrm{~mL})$ for $30 \mathrm{~min}$ at room temperature. Solvents were evaporated and coevaporated with toluene $(3 \times 5$ $\mathrm{mL}$ ). The residue was purified by silica gel flash column chromatography (gradient eluent EtOAc:cyclohexane (1:8) to (1:3)) to afford 22 (142 $\mathrm{mg}, 84 \%)$ as a white foam. 
$\mathrm{R}_{\mathrm{f}}($ EtOAc:cyclohexane $(1: 4))=0.21 ;{ }^{1} \mathrm{H}-\mathrm{NMR}\left(300 \mathrm{MHz}, \mathrm{CDCl}_{3}\right): 7.36-7.21$ (m, 20H), 5.79 (dddd, $J=17.1,10.3,6.6,6.6 \mathrm{~Hz}, 1 \mathrm{H}), 5.08-4.93(\mathrm{~m}, 4 \mathrm{H}), 4.83-4.75(\mathrm{~m}, 6 \mathrm{H}), 4.62(\mathrm{~d}, J=11.0$ $\mathrm{Hz}, 1 \mathrm{H}), 4.58(\mathrm{~d}, J=11.0 \mathrm{~Hz}, 1 \mathrm{H}), 4.53(\mathrm{~d}, J=7.8 \mathrm{~Hz}, 1 \mathrm{H}), 4.35(\mathrm{~d}, J=8.1 \mathrm{~Hz}, 1 \mathrm{H}), 3.95-3.61$ $(\mathrm{m}, 8 \mathrm{H}), 3.56-3.35(\mathrm{~m}, 4 \mathrm{H}), 2.14-1.99(\mathrm{~m}, 3 \mathrm{H}), 1.71-1.56(\mathrm{~m}, 2 \mathrm{H}), 1.19(\mathrm{~s}, 9 \mathrm{H}), 1.18(\mathrm{~s}, 9 \mathrm{H})$;

${ }^{13} \mathrm{C}-\mathrm{NMR}\left(75 \mathrm{MHz}, \mathrm{CDCl}_{3}\right)$ : 176.6, 137.9, 137.8, 128.4, 128.3, 128.0, 127.9, 127.6, 127.3, 127.3, $114.8,101.1,100.9,83.0,77.8,75.5,75.3,74.9,72.8,69.0,68.1,61.9,38.7,29.9,28.7,27.0$; $[\alpha]_{\mathrm{D}}\left(\mathrm{CHCl}_{3}, \mathrm{c}=1\right):-14.8^{\circ} ; \mathrm{IR}\left(\mathrm{CHCl}_{3}\right): 3432,3067,3008,2973,2874,1737,1603,1498,1479$, 1454, 1397, 1363, 1278, 1139, 1086, 931, 914, 886; HRMS(MALDI) calcd for $\mathrm{C}_{55} \mathrm{H}_{70} \mathrm{O}_{13} \mathrm{Na}$ 961.4709, found 961.4692.

Pent-4-en-1-yl 3,4-di- $O$-benzyl-6- $O$-(fluorenylmethoxycarbonyl)-2- $O$-pivaloyl- $\beta$-Dglucopyranosyl-( $1 \rightarrow 6)-3,4-$ di- $O$-benzyl-2- $O$-pivaloyl- $\beta$-D-glucopyranosyl-( $1 \rightarrow 6)-3,4-$ di- $O$ benzyl-2-O-pivaloyl- $\beta$-D-glucopyranoside (23). General procedure for glycosylation in solution using 22 (105 mg, $0.112 \mathrm{mmol}), 2$ (125 mg, $0.145 \mathrm{mmol})$ and TMSOTf (26 $\mu \mathrm{L}, 0.145 \mathrm{mmol})$ in $\mathrm{CH}_{2} \mathrm{Cl}_{2}(2.3 \mathrm{~mL})$ between $-30{ }^{\circ} \mathrm{C}$ and $-20{ }^{\circ} \mathrm{C}$ for $30 \mathrm{~min}$. The reaction was quenched with pyridine $(150 \mu \mathrm{L})$. Silica gel flash column chromatography (gradient eluent EtOAc/cyclohexane (1:9) to (1:5)) afforded $23(172 \mathrm{mg}, 92 \%)$ as a white foam. $\mathrm{R}_{\mathrm{f}}($ EtOAc/cyclohexane $(1: 4))=0.45$; ${ }^{1} \mathrm{H}-\mathrm{NMR}\left(300 \mathrm{MHz}, \mathrm{CDCl}_{3}\right): 7.75(\mathrm{bd}, J=7.5 \mathrm{~Hz}, 2 \mathrm{H}), 7.65-7.56(\mathrm{~m}, 2 \mathrm{H}), 7.43$ - $7.20(\mathrm{~m}$, 34H), 5.82 (dddd, $J=17.1,10.3,6.5,6.5 \mathrm{~Hz}, 1 \mathrm{H}), 5.18-4.96(\mathrm{~m}, 5 \mathrm{H}), 4.84-4.18(\mathrm{~m}, 20 \mathrm{H}), 4.08$ - $3.96(\mathrm{~m}, 2 \mathrm{H}), 3.88(\mathrm{ddd}, J=9.7,6.2,6.2 \mathrm{~Hz}, 1 \mathrm{H}), 3.80-3.40(\mathrm{~m}, 12 \mathrm{H}), 2.17$ - $2.07(\mathrm{~m}, 2 \mathrm{H})$, 1.68 (b.quint., $J \approx 7 \mathrm{~Hz}, 2 \mathrm{H}), 1.22(\mathrm{~s}, 9 \mathrm{H}), 1.22(\mathrm{~s}, 9 \mathrm{H}), 1.20(\mathrm{~s}, 9 \mathrm{H}) ;{ }^{13} \mathrm{C}-\mathrm{NMR}(75 \mathrm{MHz}$, $\left.\mathrm{CDCl}_{3}\right): 176.5,176.4,154.8,143.3,143.1,141.1,138.0,137.8,137.8,137.5,128.4,128.3,128.2$, $128.0,127.9,127.8,127.6,127.5,127.3,127.2,127.1,125.2,125.1,120.0,114.8,101.0,100.8$, $110.7,83.2,78.3,77.9,77.3,75.4,75.0,74.8,74.7,73.2,73.0,72.8,70.0,68.9,68.0,67.4,66.6$, 
46.7, 39.9, 30.2, 28.9, 27.3, $27.3 ;[\alpha]_{\mathrm{D}}\left(\mathrm{CHCl}_{3}, \mathrm{c}=0.5\right):-9.9^{\circ}$; IR $\left(\mathrm{CHCl}_{3}\right): 3067,3008,2972$, 2874, 1739, 1603, 1497, 1479, 1453, 1397, 1364, 1262, 1139, 1089; HRMS(MALDI) calcd for $\mathrm{C}_{95} \mathrm{H}_{110} \mathrm{O}_{21} \mathrm{Na} 1609.7432$, found 1609.7395.

\section{$\underline{\text { References }}$}

[1] Carrel, F.; Seeberger, P.H.; J. Carbohydr. Chem. 2007, 26, 125-139 